\title{
Co-expression network analysis of Down's syndrome based on microarray data
}

\author{
JIANPING ZHAO $^{1 *}$, ZHENGGUO ZHANG $^{2 *}$, SHUMIN REN $^{3}$, YANAN ZONG $^{3}$ and XIANGDONG KONG \\ ${ }^{1}$ Clinical Laboratory, Women and Infants Hospital of Zhengzhou, Zhengzhou, Henan 450012; \\ ${ }^{2}$ Clinical Laboratory, Henan Provincial Hospital of Traditional Chinese Medicine, Zhengzhou, Henan 450002; \\ ${ }^{3}$ Center of Prenatal Diagnosis, The First Affiliated Hospital of Zhengzhou University, Zhengzhou, Henan 450052, P.R. China
}

Received February 28, 2015; Accepted April 11, 2016

DOI: $10.3892 /$ etm.2016.3462

\begin{abstract}
Down's syndrome (DS) is a type of chromosome disease. The present study aimed to explore the underlying molecular mechanisms of DS. GSE5390 microarray data downloaded from the gene expression omnibus database was used to identify differentially expressed genes (DEGs) in DS. Pathway enrichment analysis of the DEGs was performed, followed by co-expression network construction. Significant differential modules were mined by mutual information, followed by functional analysis. The accuracy of sample classification for the significant differential modules of DEGs was evaluated by leave-one-out cross-validation. A total of 997 DEGs, including 638 upregulated and 359 downregulated genes, were identified. Upregulated DEGs were enriched in 15 pathways, such as cell adhesion molecules, whereas downregulated DEGs were enriched in maturity onset diabetes of the young. Three significant differential modules with the highest discriminative scores (mutual information>0.35) were selected from a co-expression network. The classification accuracy of GSE16677 expression profile samples was $54.55 \%$ and $72.73 \%$ when characterized by 12 DEGs and 3 significant differential modules, respectively. Genes in
\end{abstract}

Correspondence to: Mr. Xiangdong Kong, Center of Prenatal Diagnosis, The First Affiliated Hospital of Zhengzhou University, 1 Jiangshe Donglu, Zhengzhou, Henan 450052, P.R. China

E-mail: xiangdongkongxdk@163.com

"Contributed equally

Abbreviations: APP, amyloid precursor protein; DAVID, database for annotation, visualization and integrated discovery; DGEs, differentially expressed genes; DS, Down's syndrome; DSCR, Down's syndrome critical region; EST, erythroblast transformation-specific; GEO, gene expression omnibus; OLIG1, oligodendrocyte transcription factor 1; SAM, significant analysis of microarray; SVMs, support vector machines

Key words: Down's syndrome, differentially expressed genes, significant differential module, classification accuracy, co-expressed network significant differential modules were significantly enriched in 5 functions, including the endoplasmic reticulum $(\mathrm{P}=0.018)$ and regulation of apoptosis $(\mathrm{P}=0.061)$. The identified DEGs, in particular the $12 \mathrm{DEGs}$ in the significant differential modules, such as B-cell lymphoma 2-associated transcription factor 1, heat shock protein $90 \mathrm{kDa}$ beta member 1 , UBX domain-containing protein 2 and transmembrane protein 50B, may serve important roles in the pathogenesis of DS.

\section{Introduction}

Down's syndrome (DS), also known as trisomy 21, is a genetic disorder caused by trisomy of part of, or all of, chromosome 21, and is associated with significant intellectual disability, physical growth delays and slanted eyes $(1,2)$. DS may cause other complications, such as autism, coeliac disease, hypothyroidism and leukemia (3-5). Over the last several decades, numerous genes on trisomy 21 have been suggested to be associated with DS (6).

Modern genetic pathology states the hypothesis that phenotypes of DS are determined by an extra copy of one or several genes, such as oligodendrocyte transcription factor 1 present on human chromosome 21 (Hsa21) (7). A previous study demonstrated that sorting nexin family member 27, which is suppressed by a bioactive substance encoded on Hsa21, is upregulated in the brains of DS mice (8). The DS critical region (DSCR) is composed of 6 genes, including v-ets erythroblastosis virus E26 oncogene homolog 2 (ETS2) and ETS-related gene (ERG), which encode transcription factors (9). ERG and ETS2 belong to the E26 transformation-specific family, which contains key regulators of embryonic development (10). Numerous sensitive genes such as ubiquitin specific peptidase 16, which are associated with the reduced ubiquitination of cyclin-dependent kinase inhibitor 2A and expedited senescence in Ts65Dn fibroblasts, have been confirmed to be associated with DS $(11,12)$.

The identification of sensitive genes on Hsa21 has become the focus of investigations with the aim of elaborating suitable therapeutic strategies for DS, and global gene expression profiling techniques have been widely used to analyze the molecular mechanisms underlying trisomy 21 , as the expression levels of trisomic genes and those in the wider genome can be assessed (13). The majority of DS studies have been performed 
in animal models, such as the Ts65Dn mouse model of DS, which can be used to analyze the association between hippocampal pathways and cognitive dysfunction (14-16). Various samples, such as fibroblasts, whole blood, T cells and placenta, have been used to investigate the pathogenesis of DS (17-20). The samples used in the present study are derived from the brain tissue samples of patients with DS. Lockstone et al (21) screened differentially expressed genes (DEGs) in DS and performed functional analysis, and investigated significant differential modules from a co-expressed network (21).

In the present study, GSE5390 and GSE16677 were downloaded from the Gene Expression Omnibus (GEO) database, DEGs were screened for pathway enrichment analysis, then a co-expressed network was constructed and screened for significant differential modules. In addition, the classification accuracy of GSE16677 expression profile samples were evaluated, characterized by the value of average expressed DEGs and all DEGs in significant differential modules. Finally, functional enrichment analysis of DEGs in significant differential modules was performed.

\section{Materials and methods}

Microarray data. The transcription profiles of GSE5390 and GSE16677 were acquired from the GEO database (http://www. ncbi.nlm.nih.gov/geo/) (22,23). The platform names were GPL96 Affymetrix Human Genome U133A Array and GPL570 Affymetrix Human Genome U133 plus 2.0 Array, respectively. A total of 15 samples from GSE5390 were studied, including 8 normal samples and 7 DS samples of human adult brain tissue (dorsolateral prefrontal cortex) from healthy controls and individuals with DS (23). A total of 11 samples from GSE16677 were studied, including 5 normal samples and 6 DS samples of megakaryocytic leukemia blasts from patients with DS-acute megakaryoblastic leukemia (AMKL) or French-American-British M7 leukemia, and patients with non-DS-AMKL. The raw data were downloaded for further analysis. The present study was approved by the Ethics Committee of the First Affiliated Hospital of Zhengzhou University (Zhengzhou, China) and performed in accordance with ethical standards $(22,23)$.

DEG analysis. Gene expression data from GSE5390 were processed through background correction, quantile normalization and probe summarization using the robust multi-array average algorithm in the oligo software package (version 1.8.3) in Bioconductor (https://bioconductor.org/packages/release/bioc/html/oligo.html). The probe in the matrix file corresponded to the gene symbol based on the downloaded platform annotation files. The average value of the gene symbol with multiple probes was calculated. Significant Analysis of Microarray approach in the R package samr (version 2.0) (24) was used to screen the DEGs in DS samples by assigning a score based on the change in gene expression relative to the standard deviation of the repeated measurements. Corrected $\mathrm{P}<0.05$ was considered to indicate a statistically significant difference.

Pathway enrichment analysis. The Database for Annotation, Visualization and Integrated Discovery (DAVID) provides analytical tools for extracting biological meaning from large lists of genes $(25,26)$, and was used for pathway enrichment analysis of upregulated and downregulated DEGs in the current study.

Co-expressed network construction. Gene co-expression network analysis is a fusion product of microarray profile techniques and network theory, that offers an intuitive concept for illustrating the association between two genes (27). The gene expression data set GSE5390 was applied to build a co-expressed network. Cytoscape is an open software platform for visualizing complex networks and integrating these with any type of attributed data (28). The Pearson correlation coefficient is a popular coefficient for evaluating the dependence of two variables (29) and, in the present study, Pearson's coefficient analysis was performed using the Perl script program (version 5.22.1; https://www.perl.org/get.html). R>0.9 was chosen as the criterion for selecting co-expressed genes. The co-expression network was visualized using Cytoscape software (version 2.8; http://www.cytoscape.org/).

Analysis of differentially co-expressed gene modules. GraphWeb (http://biit.cs.ut.ee/graphweb/) is a public web server used to mine heterogeneous biological networks for significant function gene modules (30). The hidden Markov model (HMM) of GraphWeb can be used for modeling by defining and observing the joint probability of sequences and label sequences (31). In the present study, HMM was used for mining network modules. Mutual information (MI) between the average expression value of modules and sample labels was calculated according to the following formula (32):

$$
M I\left(\mathrm{a}^{\prime}, c\right)=\sum_{a^{\prime} \in x} \sum_{c \in y} P(x, y)=\log \frac{p(x, y)}{p(x) p(y)}
$$

In this formula, a' is the discretization average expression value; $\mathrm{c}$ is the corresponding vector of the sample label. To derive $a^{\prime}$ from $a$, activity levels are discretized into $\left[\log _{2}\right.$ (number of samples) +1$]$ values (33). Differentially co-expressed gene modules were screened with the threshold of discriminative score (defined as MI) $>0.35$. Then, functional enrichment analysis of differentially expressed genes in the differentially co-expressed gene modules was performed by DAVID. $\mathrm{P}<0.1$ was used as the cut-off criterion.

Assessment of classification efficiency. Normal and DS samples were classified by the characteristics of differentially co-expressed gene modules and differentially expressed genes. The accuracy of classification was confirmed by leave-one-out cross-validation. The leave-one-out estimate was calculated as follows (34): Each specimen was excluded from the training set one at a time and then classified on the basis of the predictor built from the data for all of the other specimens.

\section{Results}

Differentially expressed gene screening. Based on the differential expression analysis of GSE5390, a total of 997 DGEs were obtained with corrected $\mathrm{P}<0.05$, including 638 upregulated genes and 359 downregulated genes. 
Table I. Pathway enrichment analysis for upregulated and downregulated DEGs.

\begin{tabular}{|c|c|c|c|c|}
\hline DEGs & Term ID & Description & Gene no. & P-value \\
\hline \multirow[t]{15}{*}{ Upregulated } & hsa04514 & Cell adhesion molecules & 23 & $1.68 \mathrm{E}-07$ \\
\hline & hsa05416 & Viral myocarditis & 14 & 2.19E-05 \\
\hline & hsa04940 & Type I diabetes mellitus & 9 & $6.58 \mathrm{E}-04$ \\
\hline & hsa05330 & Allograft rejection & 8 & $1.26 \mathrm{E}-03$ \\
\hline & hsa04612 & Antigen processing and presentation & 12 & 1.68E-03 \\
\hline & hsa05332 & Graft-versus-host disease & 8 & $2.06 \mathrm{E}-03$ \\
\hline & hsa04142 & Lysosome & 14 & $3.34 \mathrm{E}-03$ \\
\hline & hsa05320 & Autoimmune thyroid disease & 8 & $9.57 \mathrm{E}-03$ \\
\hline & hsa00380 & Tryptophan metabolism & 7 & $1.05 \mathrm{E}-02$ \\
\hline & hsa05310 & Asthma & 6 & 1.07E-02 \\
\hline & hsa05322 & Systemic lupus erythematosus & 11 & $1.81 \mathrm{E}-02$ \\
\hline & hsa00600 & Sphingolipid metabolism & 6 & $3.54 \mathrm{E}-02$ \\
\hline & hsa00330 & Arginine and proline metabolism & 7 & $3.79 \mathrm{E}-02$ \\
\hline & hsa00071 & Fatty acid metabolism & 6 & $3.90 \mathrm{E}-02$ \\
\hline & hsa04640 & Hematopoietic cell lineage & 9 & 4.98E-02 \\
\hline \multirow[t]{2}{*}{ Downregulated } & hsa04950 & Maturity onset diabetes of the young & 4 & $1.56 \mathrm{E}-02$ \\
\hline & hsa04260 & Cardiac muscle contraction & 6 & $2.53 \mathrm{E}-02$ \\
\hline
\end{tabular}

DEGs, differentially expressed genes; ID, identification.

Pathway enrichment analysis. Pathway enrichment analysis of upregulated and downregulated DEGs was performed by DAVID. A total of 17 pathways were enriched for the DEGs (Table I). The upregulated DEGs were enriched in 15 pathways, specifically in cell adhesion molecules $\left(\mathrm{P}=1.68 \times 10^{-7}\right)$ which involved 23 genes, such as golgi glycoprotein 1 ( $G L G 1)$, human leukocyte antigen class II histocompatibility antigen (HLA-DRBI) and claudin-10. Type I diabetes mellitus $\left(\mathrm{P}=6.58 \times 10^{-4}\right)$, a complication of $\mathrm{DS}$, was enriched by 9 genes, including carboxypeptidase $\mathrm{E}$ and $H L A-D R B 1$. Downregulated DEGs were primarily enriched in two pathways: Maturity onset diabetes of the young $\left(\mathrm{P}=1.56 \times 10^{-2}\right)$ and cardiac muscle contraction $\left(\mathrm{P}=2.53 \times 10^{-2}\right)$. These 17 pathways may serve important roles in the pathogenesis of DS.

Significant differential module analysis. The constructed co-expression network included 997 nodes and 19,049 edges (Fig. 1). The co-expressed network was processed by HMM, and 36 modules were obtained. A total of 3 significant differential modules with MI>0.35 were identified (Fig. 2). Module 13 contained 5 upregulated DEGs [heterogeneous nuclear ribonucleoprotein R, B-cell lymphoma 2-associated transcription factor 1 (BCLAF1), GLG1, UBX domain-containing protein 2 (UBXD2) and transmembrane protein (TMEM) 106B] and 1 downregulated DEG (cullin 7). A total of 2 upregulated DEGs [TMEM5OB and heat shock protein $90 \mathrm{kDa}$ beta member 1 (HSP90B1)] combined with the downregulated DEG (receptor for advanced glycation end-products) combined to form module 31. Module 35 consisted of 3 downregulated DEGs, including D-beta-hydroxybutyrate dehydrogenase, solute carrier family 36 member 1 (SLC36Al) and keratin 85, type II.
Classification efficiency of significant differential modules. The accuracy of the sample classification for GSE1667 was $72.73 \%$, based on the average expression values of the 3 significant differential modules. The accuracy of the classification was $54.55 \%$ according to the expression values of all 12 DEGs in the 3 significant differential modules.

Functional enrichment analysis of significant differential modules. The functional enrichment analysis of the 12 DEGs in the 3 significant differential modules, according to the DAVID database, demonstrated that 4 genes, including SLC36A1, $U B X D 2, H S P 90 B 1$ and TMEM50B, were significantly enriched in the endoplasmic reticulum $(\mathrm{P}=0.018$; Table II). Similarly, BCLAF1, HSP90B1 and dopamine $\beta$-hydroxylase were associated with 3 functions, including the biological process of apoptosis $(\mathrm{P}=0.061)$, regulation of programmed cell death $(\mathrm{P}=0.062)$ and regulation of cell death $(\mathrm{P}=0.02)$ (Table II). Genes GLG1, UBXD2 and HSP90B1 were enriched in the endomembrane system (Table II).

\section{Discussion}

DS is a common chromosomal disorder which, to date, cannot be treated. In the present study, GSE5390 and GSE16677 were downloaded from the GEO database in order to investigate the molecular mechanisms underlying DS. A total of 997 DEGs, including 638 upregulated and 359 downregulated DEGs, were screened based on GSE5390. The DEGs in the 3 significant differential modules screened from a co-expressed network were significantly involved with the endoplasmic reticulum, endomembrane system, regulation of apoptosis, programmed cell death and cell death. Based on the pathway enrichment 
Table II. Functional enrichment analysis for the twelve DEGs in the three identified significant differential modules.

\begin{tabular}{llll}
\hline Category & \multicolumn{1}{c}{ Term } & \multicolumn{1}{c}{ Genes } \\
\hline GOTERM_CC & Endoplasmic reticulum & SLC36A1, UBXD2, HSP90B1, TMEM50B & 0.018 \\
GOTERM_BP & Regulation of apoptosis & BCLAF1, HSP90B1, DBH & 0.061 \\
GOTERM_BP & Regulation of programmed cell death & BCLAF1, HSP90B1, DBH & 0.062 \\
GOTERM_BP & Regulation of cell death & BCLAF1, HSP90B1, DBH & 0.062 \\
GOTERM_CC & Endomembrane system & GLG1, UBXD2, HSP90B1 & 0.082
\end{tabular}

DEGs, differentially expressed genes.

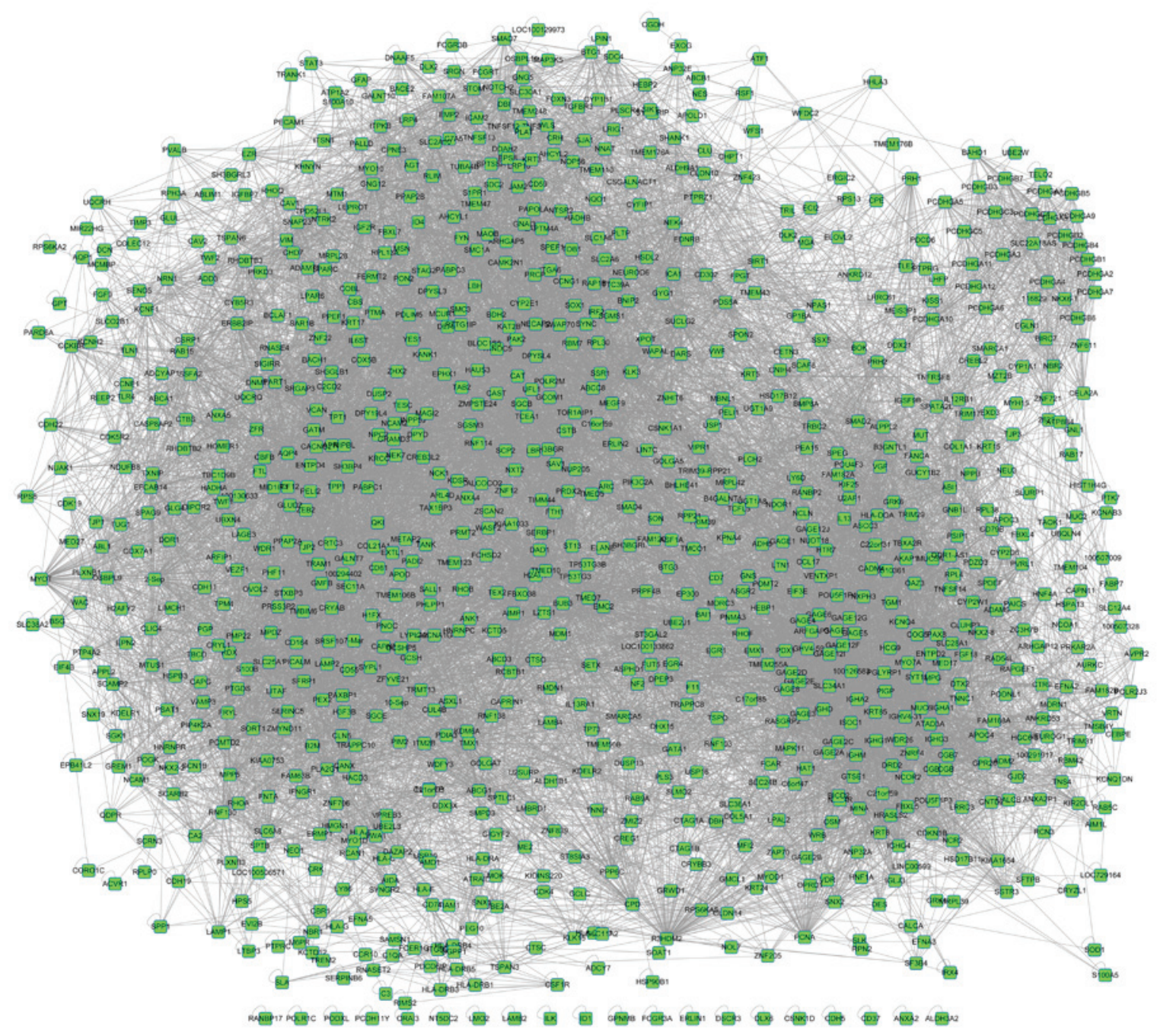

Figure 1. Co-expression network of differential expression genes. Square nodes represent significant differential genes, and lines represent the association between the genes.

A

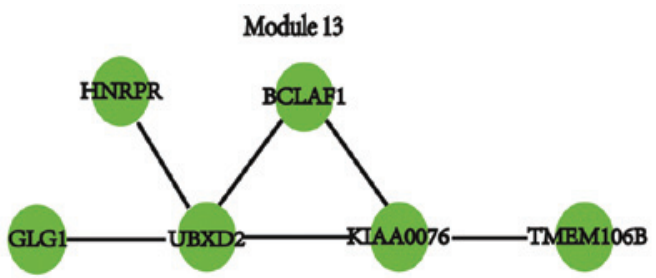

B Module 31

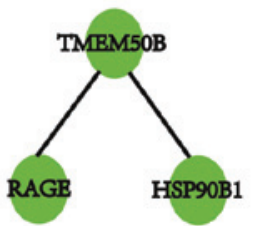

$\mathrm{C}_{\text {Module } 35}$

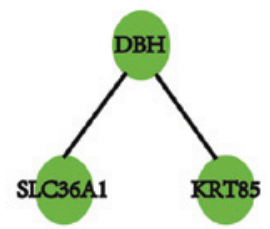

Figure 2. Significant differential modules of Down's syndrome screened from co-expressed networks using the method of mutual information. The nodes are differentially expressed genes. A, B and C indicate the three modules extracted from the network. 
analysis of DEGs, 17 significant signaling pathways involving 158 DEGs were obtained in the current study. The upregulated DEGs were significantly enriched in 15 signaling pathways, such as cell adhesion molecules, type I diabetes mellitus, autoimmune thyroid disease and lysosome. The pathway of cell adhesion molecules has previously been confirmed to be upregulated in infants with DS (35). Previous epidemiological surveys have demonstrated that the prevalence of type I diabetes mellitus in patients with DS was higher than that in the general population (36).

The present study demonstrated that 8 upregulated DEGs were enriched in the signaling pathway of autoimmune thyroid disease, of which regulator of calcineurin 1 (RCAN1) has been confirmed to contribute to the DS phenotype and autoimmune disorders $(37,38)$. RCAN1, also known as DSCR1, is located in the minimal candidate region and is expressed as four protein isoforms using four alternative first exons $(39,40)$. RCAN1 encodes a novel protein which may affect the process of transcriptional regulation or signal transduction $(39,40)$. Fuentes et al (41) demonstrated that the expression of the calcineurin-dependent gene was downregulated following the inhibition of nuclear factor of activated T-cell gene translocation to the nucleus by the overexpressed DSCR 1 . In addition, the lysosome was an important pathway enriched by 14 upregulated DEGs. A study by Kalanj-Bognar et al (42) showed that the activities of some lysosomal enzymes participated in the metabolism of sulfatides and gangliosides, and that there was a significant increase of $\beta$-galactosidase activity in DS leukocytes compared with controls.

In the current study, significant differential modules were identified from the co-expressed network and used as characteristics for classification of DS samples in GSE16677. Current studies on genomics focus on characterization of DEGs and co-expression networks, although this is a time-consuming and costly process (43-45). In order to solve these problems, Pavesi et al applied support vector machines (SVMs) with non-linear kernels, and the average accuracy of non-linear SVMs reached $66.5 \%$ (46). However, in the present study, the classification accuracy was 54.55 and $72.73 \%$ when characterized by 12 DEGs and 3 significant differential modules, respectively. More reliable results were obtained using this method by combining significant differential modules with the assessment of classification efficiency.

A total of 7 DEGs in significant differential modules were identified as being enriched in 5 pathways by functional enrichment analysis. The pathway of apoptosis regulation was enriched by BCLAF1 (module 13), HSP90B1 (module 31) and $D B H$ (module 35 ). A previous study demonstrated that DS neurons have a defect, which may lead to mental retardation early in life and promote the metabolism of reactive oxygen species that cause neuronal apoptosis (47). In addition, HSP90B1 can help stabilize protein-folding intermediates when the endoplasmic reticulum is involved in protein folding (48). TMEM50B was found to be involved with the function of endoplasmic reticulum in the current study. The Allen brain atlas investigated by Lein et al (49) showed that TMEM5OB may be a candidate for DS brain phenotypes (49), in addition, experiments on a DS mouse model demonstrated that TMEM50B was overexpressed in the DS cerebellum, compared with a normal mouse (50). The present study identified that $U B X D 2$ was enriched in two functions: The endoplasmic reticulum and the endomembrane system. A previous study demonstrated that $U B X D 2$ was a molecular chaperone involved in various cellular processes, such as endoplasmic-reticulum-associated protein degradation, and that the overexpression of $U B X D 2$ resulted in additional immunoprecipitation, which was associated with Alzheimer's disease (51). Therefore, UBXD2 may be involved in the complications of DS. BCLAF1, which intrinsically inhibits tumor cells, was demonstrated to be enriched in three functions in the present study, including the regulation of apoptosis, programmed cell death and cell death. It has been reported that BCLAF1 participates in the $\gamma \mathrm{H} 2 \mathrm{AX}$-mediated regulation of apoptosis, DNA repair, and interacts with $\gamma \mathrm{H} 2 \mathrm{AX}$ as a tumor suppressor (52). An extra unpaired chromosome in $\mathrm{DS}$ is modified by phosphorylation of $\mathrm{H} 2 \mathrm{AX}$ (53). Therefore, $B C L A F 1$ may be involved indirectly in the pathogenesis of DS. In conclusion, the DEGs in the selected differentially co-expressed gene modules may participate in the pathogenesis of DS.

\section{Acknowledgements}

The present study was supported by a grant from the Henan Province Medical Science and Technique Foundation (grant no. 201303015).

\section{References}

1. Patterson D: Molecular genetic analysis of Down syndrome. Hum Genet 126: 195-214, 2009.

2. Weijerman ME and de Winter JP: Clinical practice. The care of children with Down syndrome. Eur J Pediatr 169: 1445-1452, 2010.

3. Lott IT and Dierssen M: Cognitive deficits and associated neurological complications in individuals with Down's syndrome. Lancet Neurol 9: 623-633, 2010.

4. Myrelid A, Gustafsson J, Ollars B and Annerén G: Growth charts for Down's syndrome from birth to 18 years of age. Arch Dis Child 87: 97-103, 2002.

5. Fong CT and Brodeur GM: Down's syndrome and leukemia: Epidemiology, genetics, cytogenetics and mechanisms of leukemogenesis. Cancer Genet Cytogenet 28: 55-76, 1987.

6. Aula P, Leisti J and von Koskull H: Partial trisomy 21. Clin Genet 4: 241-251, 1973.

7. Lana-Elola E, Watson-Scales SD, Fisher EM and Tybulewicz VL: Down syndrome: Searching for the genetic culprits. Dis Model Mech 4: 586-595, 2011.

8. Wang X, Zhao Y, Zhang X, Badie H, Zhou Y, Mu Y, Loo LS Cai L, Thompson RC, Yang B, et al: Loss of sorting nexin 27 contributes to excitatory synaptic dysfunction by modulating glutamate receptor recycling in Down's syndrome. Nat Med 19: 473-480, 2013.

9. Briggs JA, Sun J, Shepherd J, Ovchinnikov DA, Chung TL, Nayler SP, Kao LP, Morrow CA, Thakar NY, Soo SY, et al: Integration-free induced pluripotent stem cells model genetic and neural developmental features of down syndrome etiology. Stem Cells 31: 467-478, 2013

10. Di Cunto F and Berto G: Molecular pathways of Down Syndrome critical region genes, In: Down Syndrome. Dey SK (ed). InTech, Rijeka, 2013.

11. Adorno M, Sikandar S, Mitra SS, Kuo A, Nicolis Di Robilant B, Haro-Acosta V, Ouadah Y, Quarta M, Rodriguez J, Qian D, et al: Usp16 contributes to somatic stem-cell defects in Down's syndrome. Nature 501: 380-384, 2013.

12. Jiang J, Jing Y, Cost GJ, Chiang JC, Kolpa HJ, Cotton AM, Carone DM, Carone BR, Shivak DA, Guschin DY, et al: Translating dosage compensation to trisomy 21. Nature 500: 296-300, 2013.

13. Letourneau A, Santoni FA, Bonilla X, Sailani MR, Gonzalez D, Kind J, Chevalier C, Thurman R, Sandstrom RS, Hibaoui $\mathrm{Y}$, et al: Domains of genome-wide gene expression dysregulation in Down's syndrome. Nature 508: 345-350, 2014. 
14. Kleschevnikov AM, Belichenko PV, Villar AJ, Epstein CJ, Malenka RC and Mobley WC: Hippocampal long-term potentiation suppressed by increased inhibition in the Ts65Dn mouse, a genetic model of Down syndrome. J Neurosci 24: 8153-8160, 2004.

15. Potier MC, Rivals I, Mercier G, Ettwiller L, Moldrich RX, Laffaire J, Personnaz L, Rossier J and Dauphinot L: Transcriptional disruptions in Down syndrome: A case study in the Ts1Cje mouse cerebellum during post-natal development. J Neurochem 97 (Suppl 1): S104-S109, 2006.

16. Amano K, Sago H, Uchikawa C, Suzuki T, Kotliarova SE, Nukina N, Epstein CJ and Yamakawa K: Dosage-dependent over-expression of genes in the trisomic region of Ts1Cje mouse model for Down syndrome. Hum Mol Genet 13: 1333-1340, 2004

17. Li CM, Guo M, Salas M, Schupf N, Silverman W, Zigman WB Husain S, Warburton D, Thaker H and Tycko B: Cell type-specific over-expression of chromosome 21 genes in fibroblasts and fetal hearts with trisomy 21. BMC Med Genet 7: 24, 2006.

18. Tang Y, Schapiro MB, Franz DN, Patterson BJ, Hickey FJ, Schorry EK, Hopkin RJ, Wylie M, Narayan T, Glauser TA, et al: Blood expression profiles for tuberous sclerosis complex 2 , neurofibromatosis type 1, and Down's syndrome. Ann Neurol 56: 808-814, 2004

19. Giannone S, Strippoli P, Vitale L, Casadei R, Canaider S, Lenzi L, D'Addabbo P, Frabetti F, Facchin F, Farina A, et al: Gene expression profile analysis in human T lymphocytes from patients with Down Syndrome. Ann Hum Genet 68: 546-554, 2004

20. FitzPatrick DR, Ramsay J, McGill NI, Shade M, Carothers AD and Hastie ND: Transcriptome analysis of human autosomal trisomy. Hum Mol Genet 11: 3249-3256, 2002.

21. Lockstone HE, Harris LW, Swatton JE, Wayland MT, Holland AJ and Bahn S: Gene expression profiling in the adult Down syndrome brain. Genomics 90: 647-660, 2007.

22. Klusmann JH, Godinho FJ, Heitmann K, Maroz A, Koch ML, Reinhardt D, Orkin SH and Li Z: Developmental stage-specific interplay of GATA1 and IGF signaling in fetal megakaryopoiesis and leukemogenesis. Genes Dev 24: 1659-1672, 2010.

23. Lockstone HE, Harris LW, Swatton JE, Wayland MT, Holland AJ and Bahn S: Gene expression profiling in the adult Down syndrome brain. Genomics 90: 647-660, 2007.

24. Tusher VG, Tibshirani R and Chu G: Significance analysis of microarrays applied to the ionizing radiation response. Proc Natl Acad Sci USA 98: 5116-5121, 2001

25. Huang da W, Sherman BT and Lempicki RA: Systematic and integrative analysis of large gene lists using DAVID bioinformatics resources. Nat Protoc 4: 44-57, 2009.

26. Dennis G Jr, Sherman BT, Hosack DA, Yang J, Gao W, Lane HC and Lempicki RA: DAVID: Database for annotation, visualization, and integrated discovery. Genome Biol 4: P3, 2003.

27. Horvath S and Dong J: Geometric interpretation of gene coexpression network analysis. PLoS Comput Biol 4: e1000117, 2008.

28. Shannon P, Markiel A, Ozier O, Baliga NS, Wang JT, Ramage D, Amin N, Schwikowski B and Ideker T: Cytoscape: A software environment for integrated models of biomolecular interaction networks. Genome Res 13: 2498-2504, 2003.

29. Liu Y, Meng Q, Chen R, Wang J, Jiang S and Hu Y: A new method to evaluate the similarity of chromatographic fingerprints: Weighted pearson product-moment correlation coefficient. J Chromatogr Sci 42: 545-550, 2004

30. Reimand J, Tooming L, Peterson H, Adler P and Vilo J: GraphWeb: Mining heterogeneous biological networks for gene modules with functional significance. Nucleic Acids Res 36: W452-W459, 2008.

31. Choo KH, Tong JC and Zhang L: Recent applications of hidden Markov models in computational biology. Genomics Proteomics Bioinformatics 2: 84-96, 2004.

32. Chuang HY, Lee E, Liu YT, Lee D and Ideker T: Network-based classification of breast cancer metastasis. Mol Syst Biol 3: 140 , 2007.

33. Tourassi GD, Frederick ED, Markey MK and Floyd CE Jr: Application of the mutual information criterion for feature selection in computer-aided diagnosis. Med Phys 28: 2394-2402, 2001.
34. Kearns M and Ron D: Algorithmic stability and sanity-check bounds for leave-one-out cross-validation. Neural Comput 11: 1427-1453, 1999.

35. Bahn S, Mimmack M, Ryan M, Caldwell MA, Jauniaux E, Starkey M, Svendsen CN and Emson P: Neuronal target genes of the neuron-restrictive silencer factor in neurospheres derived from fetuses with Down's syndrome: A gene expression study. Lancet 359: 310-315, 2002.

36. Anwar AJ, Walker JD and Frier BM: Type 1 diabetes mellitus and Down's syndrome: Prevalence, management and diabetic complications. Diabet Med 15: 160-163, 1998.

37. Kusters MA, Verstegen RH, Gemen EF and de Vries E: Intrinsic defect of the immune system in children with Down syndrome: A review. Clin Exp Immunol 156: 189-193, 2009.

38. Pritchard MA and Martin KR: RCAN1 and its potential contribution to the Down Syndrome Phenotype, 2013.

39. Fuentes JJ, Pritchard MA, Planas AM, Bosch A, Ferrer I and Estivill X: A new human gene from the Down syndrome critical region encodes a proline-rich protein highly expressed in fetal brain and heart. Hum Mol Genet 4: 1935-1944, 1995.

40. Fuentes JJ, Pritchard MA and Estivill X: Genomic organization, alternative splicing, and expression patterns of the DSCR1 (Down syndrome candidate region 1) gene. Genomics 44: 358-361, 1997.

41. Fuentes JJ, Genescà L, Kingsbury TJ, Cunningham KW, Pérez-Riba M,Estivill X and de la Luna S: DSCR1, overexpressed in Down syndrome, is an inhibitor of calcineurin-mediated signaling pathways. Hum Mol Genet 9: 1681-1690, 2000.

42. Kalanj-Bognar S, Rundek T, Furac I, Demarin V and Cosović C: Leukocyte lysosomal enzymes in Alzheimer's disease and Down's syndrome. J Gerontol A Biol Sci Med Sci 57: B16-B21, 2002.

43. Ma XJ, Yin HJ and Chen KJ: Investigation of gene expression profiles in patients with blood stasis syndrome. Zhong Xi Yi Jie He Xue Bao 6: 355-360, 2008 (In Chinese)

44. Khan J, Wei JS, Ringnér M, Saal LH, Ladanyi M, Westermann F, Berthold F, Schwab M, Antonescu CR, Peterson C and Meltzer PS: Classification and diagnostic prediction of cancers using gene expression profiling and artificial neural networks. Nat Med 7: 673-679, 2001.

45. Liu Y: Active learning with support vector machine applied to gene expression data for cancer classification. J Chem Inf Comput Sci 44: 1936-1941, 2004.

46. Pavesi G and Valentini G: Classification of co-expressed genes from DNA regulatory regions. Information Fusion 10: 233-241, 2009.

47. Busciglio J and Yankner BA: Apoptosis and increased generation of reactive oxygen species in Down's syndrome neurons in vitro. Nature 378: 776-779, 1995.

48. Kim I, Xu W and Reed JC: Cell death and endoplasmic reticulum stress: Disease relevance and therapeutic opportunities. Nat Rev Drug Discov 7: 1013-1030, 2008.

49. Lein ES, Hawrylycz MJ, Ao N, Ayres M, Bensinger A, Bernard A, Boe AF, Boguski MS, Brockway KS, Byrnes EJ, et al: Genome-wide atlas of gene expression in the adult mouse brain. Nature 445: 168-176, 2007.

50. Moldrich RX, Lainé J, Visel A, Beart PM, Laffaire J, Rossier J and Potier MC: Transmembrane protein 50b (C21orf4), a candidate for Down syndrome neurophenotypes, encodes an intracellular membrane protein expressed in the rodent brain. Neuroscience 154: 1255-1266, 2008.

51. Liang J, Yin C, Doong H, Fang S, Peterhoff C, Nixon RA and Monteiro MJ: Characterization of erasin (UBXD2): A new ER protein that promotes ER-associated protein degradation. J Cell Sci 119: 4011-4024, 2006

52. Lee YY, Yu YB, Gunawardena HP, Xie L and Chen X: BCLAF1 is a radiation-induced $\mathrm{H} 2 \mathrm{AX}$-interacting partner involved in $\gamma \mathrm{H} 2 \mathrm{AX}$-mediated regulation of apoptosis and DNA repair. Cell Death Dis 3: e359, 2012.

53. Reinholdt LG, Czechanski A, Kamdar S, King BL, Sun F and Handel MA: Meiotic behavior of aneuploid chromatin in mouse models of Down syndrome. Chromosoma 118: 723-736, 2009. 